\title{
A silicon-rhodamine chemical-genetic hybrid for far red voltage imag- ing from defined neurons in brain slice
}

\author{
Gloria Ortiz, ${ }^{\ddagger}$ Pei Liu, ${ }^{\ddagger}$ Parker E. Deal, ${ }^{\ddagger}$ Ashley K. Nensel, ${ }^{\ddagger}$ Kayli N. Martinez,, Kiarash

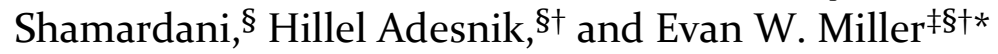

Departments of ${ }^{\ddagger}$ Chemistry and ${ }^{\S}$ Molecular \& Cell Biology and ${ }^{\dagger}$ Helen Wills Neuroscience Institute. University of California, Berkeley, California 94720, United States.

Supporting Information Placeholder

\begin{abstract}
We describe the design, synthesis, and application of voltage-sensitive silicon rhodamines. Based on the Berkeley Red Sensor of Transmembrane potential, or BeRST, scaffold, the new dyes possess an isomeric molecular wire for improved alignment in the plasma membrane and contain $\mathbf{2}^{\prime}$ carboxylic acids for ready functionalization. Conjugation with secondary amines affords tertiary amides that localize to cellular membranes and respond to voltage changes with a $24 \%$ $\Delta \mathrm{F} / \mathrm{F}$ per $100 \mathrm{mV}$. When combined with a flexible polyethyleneglycol (PEG) linker and a chloroalkane HaloTag ligand, the new indicators, or isoBeRST dyes, enable voltage imaging from genetically defined cells and neurons. Covalent ligation of isoBeRST to cell surface-expressed HaloTag enzymes provides up to 3 -fold improved labeling over previous, rhodamine-based hybrid strategies. We show that isoBeRST-Halo hybrid indicators achieve single-trial voltage imaging of membrane potential dynamics from dissociated rat hippocampal neurons or mouse cortical neurons in brain slices. With farred/near infrared excitation and emission, turn-on response to action potentials, effective cell labeling in thick tissue, and excellent photostability, the new isoBeRST-Halo derivatives provide an important complement to voltage imaging in neurobiology.
\end{abstract}

Voltage imaging in the central nervous system promises to transform the ways in which we observe brain systems..$^{1-2}$ Recently, a number of approaches to voltage imaging have emerged, including methods that rely solely on synthetic dyes $^{3-9}$ or genetically encoded proteins. ${ }^{10-17}$ Alternatively, hybrid methodologies can combine the unique properties of synthetic dyes-high molecular brightness, wide availability of colors, or fast response kinetics-with the cellular specificity of genetically encoded methods. ${ }^{18-23}$ Our group recently reported the development of a completely synthetic voltagesensitive fluorophore, Berkeley Red Sensor of Transmembrane potential 1, or BeRST 1, a silicon-rhodamine-based indicator that we hypothesize operates via voltage-sensitive photoinduced electron transfer (PeT). ${ }^{24}$ The high sensitivity $(24 \%$ $\Delta \mathrm{F} / \mathrm{F}$ per $100 \mathrm{mV}$ ), fast response kinetics, photostability, and far red/near infra-red excitation and emission profile have enabled the use of BeRST 1 in a number of voltage imaging applications. ${ }^{25-31}$

\section{Scheme 1. Overview of isoBeRST-Halo}

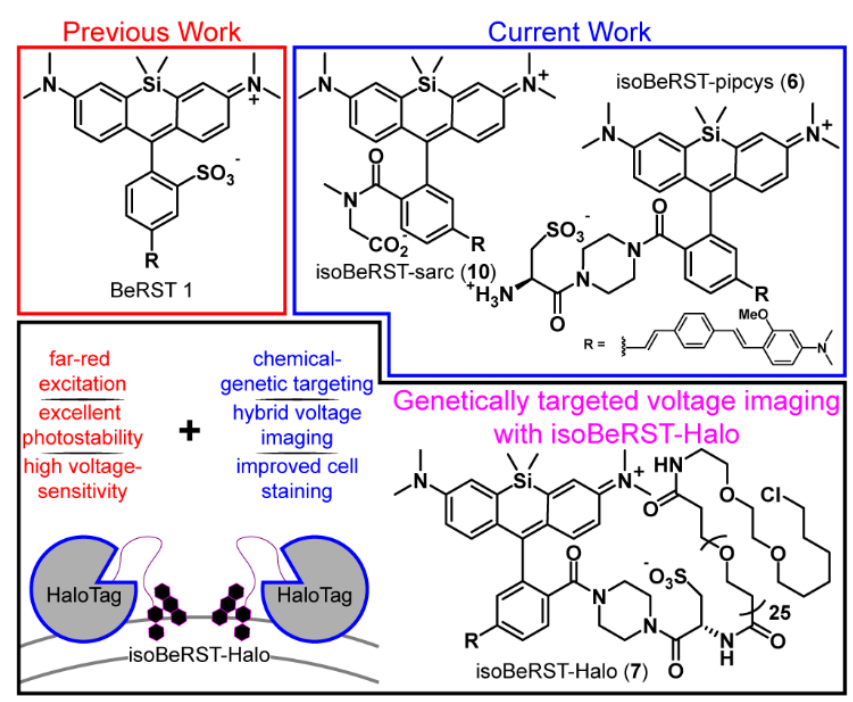

However, the use of BeRST 1 has been largely restricted to in vitro systems of homogeneous cell types. Usage in more complex settings, like thick brain tissue, remains a challenge because of a lack of methods to genetically target BeRST 1 to defined cells. Here we report two new synthetic BeRST dyes and show that this new class of indicator can be combined with a genetically-encoded protein tether to enable voltage imaging from defined cells in mouse brain slice.

To enable genetic targeting of BeRST-style dyes, we redesigned the synthesis of BeRST. We replaced the 2 '-sulfonate of BeRST with a carboxylate: this allows for addition of covalent tethers and mimics our previous design success with Rhodamine-based Voltage Reporters (RhoVRs).32-33 We also used the 5 ' version of molecular wire, since the 5 , or isomeric, version showed improved voltage sensitivity compared to the 4' RhoVR. ${ }^{32}$ Additionally, the commercial availability of the precursors to the aldehyde starting material substantially simplified the synthetic route (Scheme $\mathbf{S} \mathbf{1}$ ). The optimized synthesis of isoBeRST-sarc 10 begins with a Heck reaction between fluorophore $\mathbf{1}^{34-35}$ and (E)-3-methoxy- $N, N$-dimethyl-4-(4-vinylstyryl)aniline ${ }^{36}$ to obtain carboxy silicon rhodamine 8 (Scheme S1). Dye $\mathbf{8}$ is coupled to sarcosine tert-butyl ester using oxalyl chloride, followed 


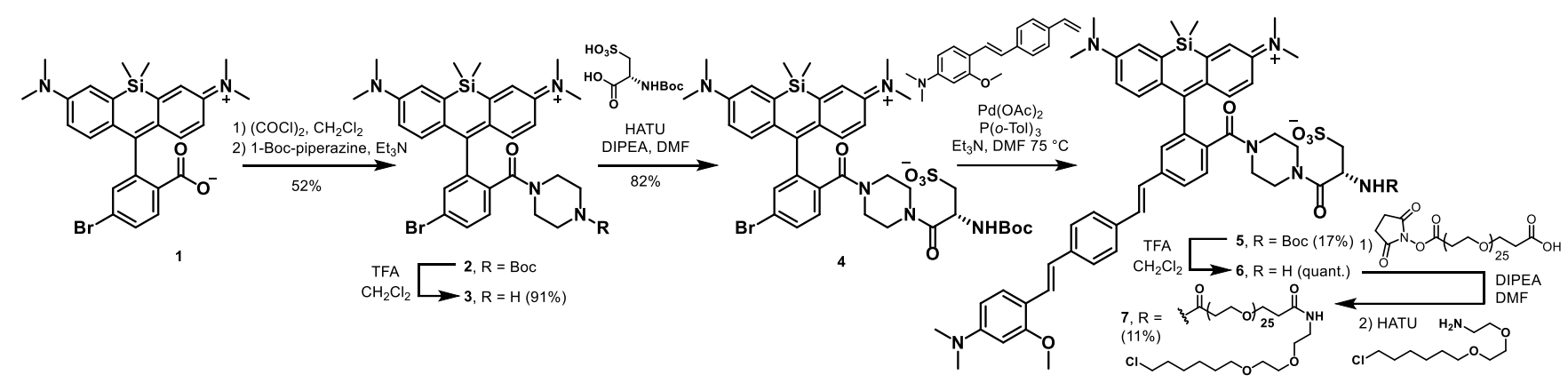

Table 1. Properties of isoBeRST indicators

\begin{tabular}{c|c|c|c|c|c|c} 
Compound & $\lambda_{\max } / \mathrm{nm}^{a}$ & $\lambda_{\mathrm{em}} / \mathrm{nm}^{a}$ & $\varepsilon$ & $\Phi^{\mathrm{a}, \mathrm{b}}$ & $\% \Delta \mathrm{F} / \mathrm{F} / 100 \mathrm{mV}^{c}$ & Relative brightness $^{d}$ \\
\hline \hline isoBeRST-pipcys 6 & 662 & 681 & 172,000 & 0.061 & $24 \pm 1.9$ & $100 \%$ \\
\hline isoBeRST-Halo 7 & 662 & 677 & - & 0.042 & $21 \pm 1.2$ & $30 \%$ \\
\hline isoBeRST-sarc 10 & 661 & 681 & 107,700 & 0.098 & $24 \pm 2.6$ & -
\end{tabular}

${ }^{a}$ In PBS, $\mathrm{pH}$ 7.4, o.1\% SDS. ${ }^{b}$ Referenced to Cy5.5-carboxylic acid in PBS. cVoltage-clamped HEK cells. Error is \pm S.D. for $\mathrm{n}=5-6$ cells. ${ }^{d}$ In HEK cells. Error is \pm S.E.M for $n=4$ coverslips (>100 cells per coverslip for relative brightness).

by a TFA-catalyzed deprotection of the tert-butyl ester to give the voltage-sensitive fluorophore isoBeRST-sarc 10, which is the Si-rhodamine analog of RhoVR $1 .{ }^{32}$

We also synthesized the piperazine-cysteic acid conjugate of isoBeRST, or isoBeRST-pipcys $\mathbf{6}$, since this configuration allowed us to target RhoVR dyes to specific cells using HaloTag (Scheme 2).33 The synthesis of isoBeRST-pipcys 6 and isoBeRST-Halo 7 follows a sequential amide-coupling / Heck coupling sequence. This provided higher overall yields than amide coupling with the assembled molecular wire/fluorophore 8 . The cyclic, piperazine-derived tertiary amide of 3 appears more stable than the amide formed from sarcosine, based on its ability to undergo successful Pd-catalyzed synthesis of isoBeRST-pipcys $\mathbf{6}$. The route begins with oxalyl chloride mediated coupling of reported silicon rhodamine $\mathbf{1}$ with $\mathbf{1 - B o c -}$ piperazine, followed by TFA deprotection to yield silicon rhodamine 2 (Scheme 2). A second coupling mediated by HATU installed Boc-L-cysteic acid, affording 3. Compound 3 was then submitted to a Heck reaction with phenylene-vinylene wire. Subsequent TFA deprotection gives voltage-sensitive fluorophore isoBeRST-pipcys 6 in $17 \%$ yield. In a one-pot sequence, isoBeRST-pipcys $\mathbf{6}$ is reacted with acid-dPEG ${ }_{25}-\mathrm{NHS}$ ester, followed by addition of HaloTag amine (Scheme $\mathbf{2}$ ) and HATU. The complete reaction was purified via preparativescale HPLC to yield the genetically-targetable isoBeRST-Halo 7 in $11 \%$ yield.

Spectroscopic characterization of isoBeRST-sarc 1o, isoBeRST-pipcys 6, and isoBeRST-Halo 7 reveals that all three voltage indicators possess similar photophysical properties (Table 1, Figures 1, S1, and S2). IsoBeRST-sarc 10 displays a $\lambda_{\max }$ at $661 \mathrm{~nm}$, similar to BeRST $1\left(\lambda_{\max }=658 \mathrm{~nm}\right)$ and identical to isoBeRST-pipcys 6 and isoBeRST-Halo 7. IsoBeRST-sarc 10 possesses an emission maximum of $681 \mathrm{~nm}$ and a quantum yield $(\Phi)$ of $9.8 \%$, while isoBeRST-pipcys 6 has an emission maximum of $681 \mathrm{~nm}$ and $\Phi$ of $6.1 \%$. IsoBeRST-Halo 7 has an emission maximum of $677 \mathrm{~nm}$ and $\Phi$ of $4.2 \%$.
All of the new Si-rhodamine indicators are voltage-sensitive. In human embryonic kidney (HEK) cells untargeted dyes isoBeRST-sarc 10 (Figure S1) and isoBeRST-pipcys 6 (Figure S2) localize to the plasma membrane and are voltage sensitive. IsoBeRST-pipcys 6 has a voltage sensitivity of $24 \% \pm 2 \% \Delta \mathrm{F} / \mathrm{F}$ per $100 \mathrm{mV}(\mathrm{SNR}=110 \pm 15)$, identical to BeRST $1(24 \% \pm 5 \%$ $\Delta \mathrm{F} / \mathrm{F}$ per $100 \mathrm{mV})^{24}$ and to isoBeRST-sarc 10 (Table 1 ). We selected isoBeRST-pipcys 6 to evaluate in neurons because of the higher yielding synthesis and stability compared to isoBeRSTsarc 1o. In cultured rat hippocampal neurons, isoBeRST-pipcys 6 (50o $\mathrm{nM}$ ) provided clear resolution of action potentials (Figure $\mathrm{S}_{3}$ ).

The genetically-targetable isoBeRST-Halo 7 selectively labels HEK cells expressing cell-surface HaloTag (Figure 1 and S4). We expressed HaloTag on the surface of mammalian cells using a fusion with a single-pass transmembrane domain. ${ }^{33} \mathrm{At}$ $500 \mathrm{nM}$ isoBeRST-Halo 7, cells expressing cell-surface HaloTag are approximately 14 -fold brighter than un-transfected control cells (Figure S4f). At lower concentrations (50 nM), fluorescence intensity in HaloTag-expressing cells increases to approximately 30 -fold over non-HaloTag expressing cells (Figure S4f). This is three times better contrast than RhoVRHalo labeling (10-15 fold). 33 Although expression levels of HaloTag vary slightly with transient transfection, a screen of isoBeRST-Halo 7 concentrations reveals that HaloTag binding sites appear to saturate at around 50 to $100 \mathrm{nM}$ (Figure $\mathbf{S}_{4} \mathbf{f}$ ). The drop in contrast ratio, from $\sim 30$-fold at $50 \mathrm{nM}$ to about $14^{-}$ fold at $500 \mathrm{nM}$ comes from a small increase in background staining in control cells (an increase of about 4 percentage points, from $5 \%$ to $9 \%)$. Importantly, isoBeRST 7 (50 nM) is voltage-sensitive, with a voltage sensitivity of $21 \% \pm 1 \% \Delta \mathrm{F} / \mathrm{F}$ per $100 \mathrm{mV}$ and an SNR of $42 \pm 7$ (Figure 1 and Table 1). IsoBeRST-Halo 7 maintains about the same voltage sensitivity as isoBeRST-pipcys 6 (500 nM), indicating that the covalently tethered dye remains properly oriented in the plasma membrane (Table 1 and Figure 1 ). 
a)

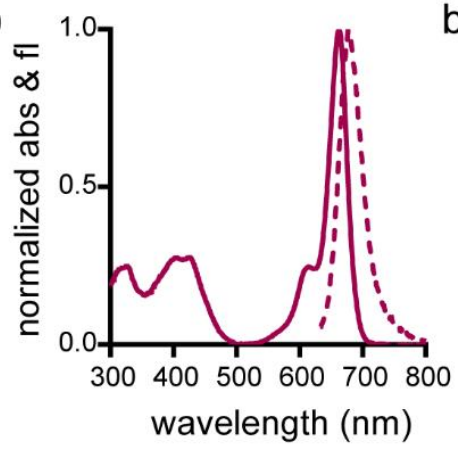

b)

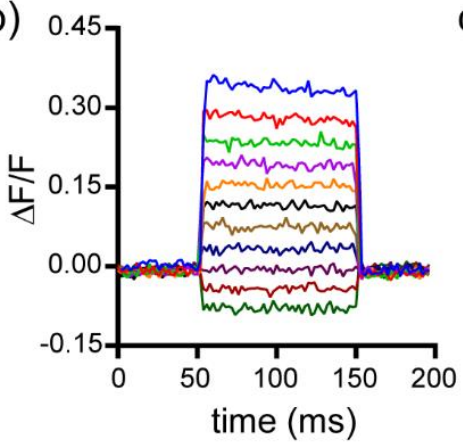

c)

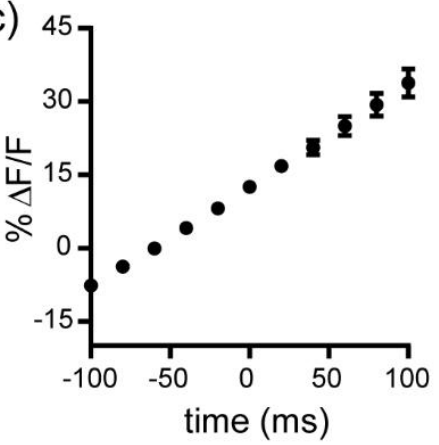

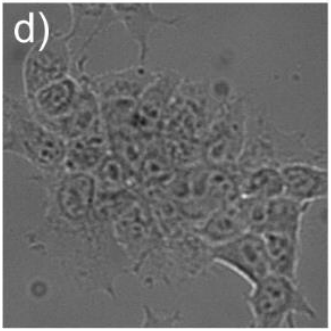
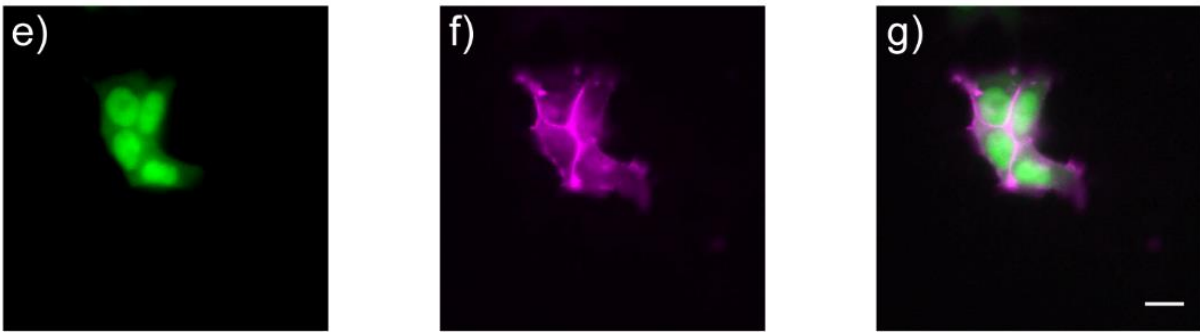

Figure 1. Cellular and in vitro characterization of isoBeRST-Halo 7. a) Normalized absorbance (solid line) and emission (dashed line) spectra of isoBeRST-Halo 7 in PBS, $\mathrm{pH} 7.4$. b) Plot of the fractional change in fluorescence of $7 \mathrm{vs}$ time for $100 \mathrm{~ms}$ hyper- and depolarizing steps $( \pm 100 \mathrm{mV}$ in $20 \mathrm{mV}$ increments) from a holding potential of $-60 \mathrm{mV}$ for single HEK cells under whole-cell voltage-clamp mode. c) Plot of $\% \Delta \mathrm{F} / \mathrm{F}$ vs final membrane potential. Data are mean \pm S.D. for $\mathrm{n}=6$ cells. d-g) Wide-field microscopy images of HEK cells transfected with CMV-HaloTag-pDisplay and stained with isoBeRST-Halo 7 (50 nM, $30 \mathrm{mins}$ ). d) DIC image of HEK cells. e) Nuclear EGFP fluorescence indicates HaloTag expression. f) isoBeRST-Halo fluorescence. g) Merge of fluorescence from EGFP (green) and isoBeRST-Halo (magenta). Scale bar is $10 \mu \mathrm{m}$.

Covalently-tethered isoBeRST-Halo 7 visualizes voltage changes in genetically-defined neurons. Dissociated, cultured rat hippocampal neurons transfected with HaloTag under control of the synapsin promotor (to drive neuron-specific expression) were labeled with $50 \mathrm{nM}$ isoBeRST-Halo. Neurons expressing HaloTag show excellent selectivity, revealing good localization of the dye to the outer membrane (Figure 2 and $\mathbf{S}_{5}$ ). The best contrast between HaloTag-expressing and control cells is achieved using $50 \mathrm{nM}$ isoBeRST-Halo (50x brighter than untransfected cells) when compared to $100 \mathrm{nM}$ isoBeRST-Halo (3ox brighter than untransfected cells). High isoBeRST-Halo fluorescence correlates with high levels of HaloTag/GFP (Figure S5e-g). Using these optimized loading conditions, we demonstrated the ability to record spontaneous and evoked activity in neurons (Figure se and Figure S6). IsoBeRST-Halo responded to field stimulated evoked action potentials with a $10 \% \pm 0.3 \% \Delta \mathrm{F} / \mathrm{F}$ and SNR of $15 \pm 1$ (19 cells).

We next evaluated the ability of isoBeRST-Halo 7 to monitor voltage dynamics from neurons in brain slice. We introduced genes for HaloTag and a co-expression marker, blue fluorescent protein, or BFP, on separate plasmids via in utero electroporation in mouse embryos. 37 We prepared tissue slices from the brains of these mice and stained the slices with isoBeRST-Halo 7 (250 to $500 \mathrm{nM}, 15 \mathrm{~min}$ ). Confocal fluorescence microscopy reveals localization of isoBeRST-Halo fluorescence in the cell membranes of neurons that express BFP and HaloTag (Figure 3a,b). Both cell bodies and more distal processes like axonal and dendritic membranes appear fluorescent (Figure $\mathbf{3} \mathbf{a}, \mathbf{b}$ and Figure $\mathbf{S}_{\mathbf{7}}$ ), mirroring results in dissociated rat neurons (Figure 2). Unlabeled
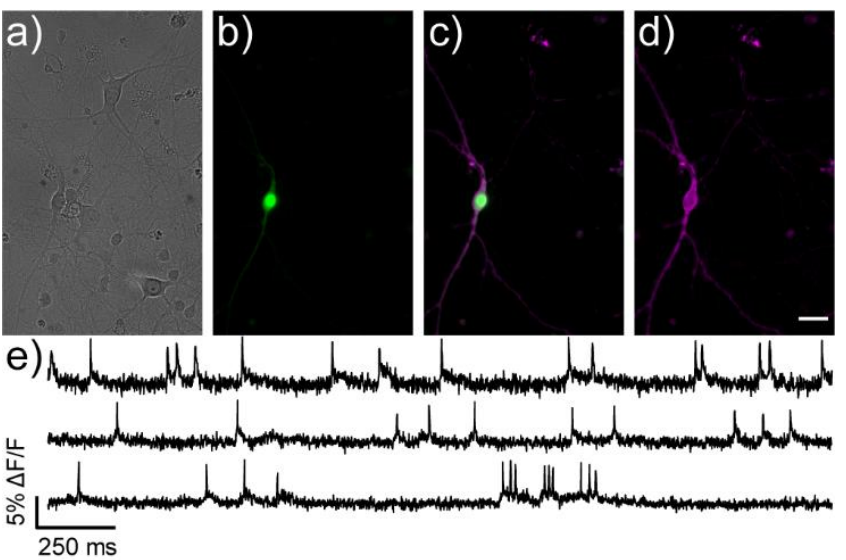

Figure 2. Monitoring spontaneous activity in neurons with isoBeRST-Halo 7. a-d) Wide-field microscopy images of isoBeRST-Halo in a HaloTag-expressing neuron. a) DIC image of neurons. b) Nuclear EGFP fluorescence indicates HaloTag expression. c) Merge of EGFP (green) and isoBeRST-Halo (magenta) fluorescence. d) isoBeRST-Halo fluorescence is restricted to the membrane. Scale bar is $20 \mu \mathrm{m}$. e) Optical recordings at $500 \mathrm{~Hz}\left(1.94 \mathrm{~W} / \mathrm{cm}^{2}\right)$ of spontaneous activity shown as $\Delta \mathrm{F} / \mathrm{F}$ vs time for HaloTag-expressing neurons from different coverslips labeled with 7 . 

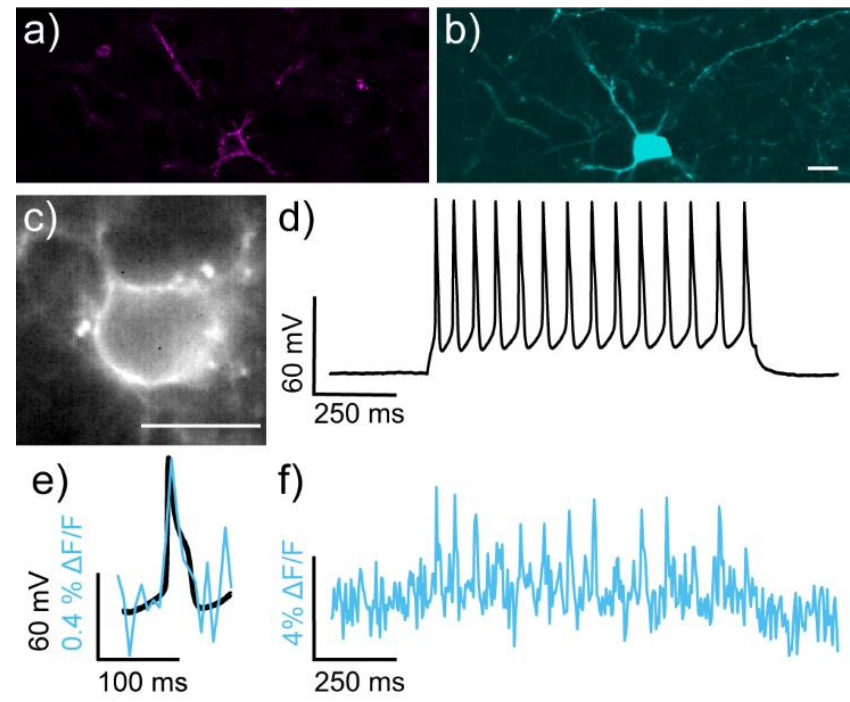

Figure 3. Characterization of isoBeRST-Halo in mouse brain slice expressing HaloTag-pDisplay and pCAG-BFP. a-b) Confocal microscopy images of a HaloTag-expressing neuron stained with a) isoBeRST-Halo (50o $\mathrm{nM}, 30 \mathrm{~min}, 23^{\circ} \mathrm{C}$ ) and expressing b) BFP. Scale bar is $20 \mu \mathrm{m}$. c) Wide-field microscopy image of isoBeRST-Halo stained slice acquired during patch-clamp electrophysiology. d) Plot of voltage vs. time for cell in panel (c). e) Overlay of membrane potential (black) and isoBeRST-Halo fluorescence (teal). f) Plot of $\Delta \mathrm{F} / \mathrm{F}$ fluorescence from isoBeRST-Halo fluorescence for the cell in panel (c). The $\Delta \mathrm{F} / \mathrm{F}$ trace was acquired at $0.5 \mathrm{kHz}$ and represents single-trial acquisition.

cell bodies appear as dark spots, indicating that labeling of neurons requires HaloTag expression (Figure $\mathbf{S}_{7}$ ). In mouse brain slices, isoBeRST-Halo is voltage-sensitive. Simultaneous patch clamp electrophysiology and fluorescence imaging establishes that isoBeRST-Halo tracks action potentials in a single trial (Figure $\mathbf{3 c}$-f). The voltage-sensitive fluorescence of isoBeRST-Halo corresponds well with the electrode-based recording of action potentials (Figure $3 \mathbf{e}$ ). IsoBeRST-Halo 7 detects action potentials (Figure 3 f) with a SNR of $4.9 \pm 1.3$ (S.D., $\mathrm{n}=10$ spikes) and a $\Delta \mathrm{F} / \mathrm{F}$ of $3.3 \% \pm 0.6 \%$ (S.D., $\mathrm{n}=10$ spikes). The sensitivity of isoBeRST-Halo 7 in brain slices compares favorably to RhoVR-Halo, which has a higher voltage sensitivity (34\%) than isoBeRST-Halo (21\%) in HEK cells and has an SNR of 3.3 and a $\Delta \mathrm{F} / \mathrm{F}$ of $4.3 \%$ in brain slice. 33

In summary, we describe the design, synthesis, and application of a new silicon-rhodamine-based voltage-sensitive fluorophores. The new BeRST derivatives rely on a 2' carboxylate, rather than sulfonate, and can be combined with secondary amines to generate tertiary amides that function well as voltage indicators in their own right, with $\Delta \mathrm{F} / \mathrm{F}$ values matching the sulfonated-based BeRST 1. ${ }^{24}$ Unlike BeRST 1, however, the new carboxy-containing isoBeRST derivatives reported here can be readily incorporated into a hybrid genetic targeting framework. When combined in this way, isoBeRST-Halo (7) enables selective labeling of cells expressing cell-surface HaloTag, including HEK293T cells, dissociated rat hippocampal neurons, and cortical neurons in mouse brain slices. Labeling with isoBeRST-Halo provides improved contrast between HaloTag-expressing and non-expressing cells, compared to RhoVR1-Halo. 33 In all of these contexts, isoBeRST-Halo is voltage-sensitive, with $\Delta \mathrm{F} / \mathrm{F}$ values comparable to the parent BeRST 1 indicator.
Voltage imaging with isoBeRST-Halo (7) provides an important complement to voltage imaging efforts. It offers a turn-on indicator for action potentials, possesses an excitation spectrum aligned with common excitation sources, operates in the far-red / near infrared, and takes advantage of the high photostability of silicon-rhodamines. ${ }^{24}$ In the future, we will maximize expression of cell-surface HaloTag, since one limitation of the covalent tethering approach is that the stoichiometric labeling limits the number of indicators that can be added to a cell membrane. Finally, we envision that isoBeRSTHalo can pair with optically 33 and enzymatically orthogonal hybrid genetic labeling strategies $3^{8-40}$ to provide multi-color voltage imaging in complex tissues.

\section{Supporting Information}

Supplementary data, including supporting figures, spectra, procedures, and analysis. This material is available free of charge via the Internet at http://pubs.acs.org.

\section{Corresponding Author}

*Evan W. Miller, evanwmiller@berkeley.edu

\section{ACKNOWLEDGMENT}

Research in the Miller lab is supported by grants from the NIH (Ro1NSo98088) and Klingenstein-Simon Foundations (40746). E.W.M and H.A. acknowledge support from NSF Neuronex (1707350). G.O. was supported by a Gilliam Research Fellowship from Howard Hughes Medical Institute. P.L. was supported by a graduate fellowship from A*STAR. K.N.M. was supported in part by a training grant from the NIH (T32GMo66698). Confocal imaging experiments were performed at the CRL Molecular Imaging Center, supported by the Helen Wills Neuroscience Institute. HRMS data were collected at the $\mathrm{QB}_{3} /$ Chemistry Mass Spectrometry Facility (UC Berkeley) with the assistance of Dr. Ulla N. Andersen.

\section{REFERENCES}

1. Peterka, D. S.; Takahashi, H.; Yuste, R., Imaging Voltage in Neurons. Neuron 2011, 69 (1), 9-21.

2. Scanziani, M.; Häusser, M., Electrophysiology in the age of light. Nature 2009, 461 (7266), 930-939.

3. Liu, P.; Miller, E. W., Electrophysiology, Unplugged: Imaging Membrane Potential with Fluorescent Indicators. Accounts of Chemical Research 2020, 53 (1), 11-19.

4. $\quad$ Sayresmith, N. A.; Saminathan, A.; Sailer, J. K.; Patberg, S. M.; Sandor, K.; Krishnan, Y.; Walter, M. G., Photostable VoltageSensitive Dyes Based on Simple, Solvatofluorochromic, Asymmetric Thiazolothiazoles. Journal of the American Chemical Society 2019, 141 (47), 18780-1879o.

5. Reeve, J. E.; Corbett, A. D.; Boczarow, I.; Kaluza, W.; Barford, W.; Bayley, H.; Wilson, T.; Anderson, H. L., Porphyrins for Probing Electrical Potential Across Lipid Bilayer Membranes by Second Harmonic Generation. Angewandte Chemie International Edition 2013, 52 (34), 9044-9048.

6. Rowland, C. E.; Susumu, K.; Stewart, M. H.; Oh, E.; Mäkinen, A. J.; O'Shaughnessy, T. J.; Kushto, G.; Wolak, M. A.; Erickson, J. S.; L. Efros, A.; Huston, A. L.; Delehanty, J. B., Electric Field Modulation of Semiconductor Quantum Dot Photoluminescence: Insights Into the Design of Robust Voltage-Sensitive Cellular Imaging Probes. Nano Letters 2015, 15 (10), 6848-6854.

7. Yan, P.; Acker, C. D.; Zhou, W. L.; Lee, P.; Bollensdorff, C.; Negrean, A.; Lotti, J.; Sacconi, L.; Antic, S. D.; Kohl, P.; Mansvelder, H. D.; Pavone, F. S.; Loew, L. M., Palette of fluorinated voltage-sensitive hemicyanine dyes. Proceedings of the National Academy of Sciences of the United States of America 2012, 109 (50), 20443-8.

8. Miller, E. W.; Lin, J. Y.; Frady, E. P.; Steinbach, P. A.; Kristan, W. B.; Tsien, R. Y., Optically monitoring voltage in neurons by photo- 
induced electron transfer through molecular wires. Proceedings of the National Academy of Sciences 2012, 109 (6), 2114-2119.

9. $\quad$ Treger, J. S.; Priest, M. F.; Iezzi, R.; Bezanilla, F., Real-time imaging of electrical signals with an infrared FDA-approved dye. Biophysical journal 2014, 107 (6), Lo9-12.

10. Piatkevich, K. D.; Jung, E. E.; Straub, C.; Linghu, C.; Park, D.; Suk, H. J.; Hochbaum, D. R.; Goodwin, D.; Pnevmatikakis, E.; Pak, N.; Kawashima, T.; Yang, C. T.; Rhoades, J. L.; Shemesh, O.; Asano, S.; Yoon, Y. G.; Freifeld, L.; Saulnier, J. L.; Riegler, C.; Engert, F.; Hughes, T.; Drobizhev, M.; Szabo, B.; Ahrens, M. B.; Flavell, S. W.; Sabatini, B. L.; Boyden, E. S., A robotic multidimensional directed evolution approach applied to fluorescent voltage reporters. Nature chemical biology 2018, 14 (4), 352-360.

11. Hochbaum, D. R.; Zhao, Y.; Farhi, S. L.; Klapoetke, N.; Werley, C. A.; Kapoor, V.; Zou, P.; Kralj, J. M.; Maclaurin, D.; Smedemark-Margulies, N.; Saulnier, J. L.; Boulting, G. L.; Straub, C.; Cho, Y. K.; Melkonian, M.; Wong, G. K.; Harrison, D. J.; Murthy, V. N.; Sabatini, B. L.; Boyden, E. S.; Campbell, R. E.; Cohen, A. E., All-optical electrophysiology in mammalian neurons using engineered microbial rhodopsins. Nature methods 2014, 11 (8), 825-33.

12. Kannan, M.; Vasan, G.; Huang, C.; Haziza, S.; Li, J. Z.; Inan, H.; Schnitzer, M. J.; Pieribone, V. A., Fast, in vivo voltage imaging using a red fluorescent indicator. Nature methods 2018, 15 (12), 11081116.

13. Abdelfattah, A. S.; Farhi, S. L.; Zhao, Y.; Brinks, D.; Zou, P.; Ruangkittisakul, A.; Platisa, J.; Pieribone, V. A.; Ballanyi, K.; Cohen, A. E.; Campbell, R. E., A Bright and Fast Red Fluorescent Protein Voltage Indicator That Reports Neuronal Activity in Organotypic Brain Slices. The Journal of neuroscience : the official journal of the Society for Neuroscience 2016, 36 (8), 2458-72.

14. Jin, L.; Han, Z.; Platisa, J.; Wooltorton, Julian R. A.; Cohen, Lawrence B.; Pieribone, Vincent A., Single Action Potentials and Subthreshold Electrical Events Imaged in Neurons with a Fluorescent Protein Voltage Probe. Neuron 2012, 75 (5), 779-785.

15. Gong, Y.; Huang, C.; Li, J. Z.; Grewe, B. F.; Zhang, Y.; Eismann, S.; Schnitzer, M. J., High-speed recording of neural spikes in awake mice and flies with a fluorescent voltage sensor. Science (New York, N.Y.) 2015, 350 (6266), 1361-6.

16. Lin, M. Z.; Schnitzer, M. J., Genetically encoded indicators of neuronal activity. Nature neuroscience 2016, 19 (9), 1142-53.

17. Wu, J.; Liang, Y.; Chen, S.; Hsu, C.-L.; Chavarha, M.; Evans, S. W.; Shi, D.; Lin, M. Z.; Tsia, K. K.; Ji, N., Kilohertz two-photon fluorescence microscopy imaging of neural activity in vivo. Nature methods 2020, 17 (3), 287-290.

18. Chanda, B.; Blunck, R.; Faria, L. C.; Schweizer, F. E.; Mody, I.; Bezanilla, F., A hybrid approach to measuring electrical activity in genetically specified neurons. Nature neuroscience 2005, 8 (11), 161926.

19. Ghitani, N.; Bayguinov, P. O.; Ma, Y.; Jackson, M. B., Singletrial imaging of spikes and synaptic potentials in single neurons in brain slices with genetically encoded hybrid voltage sensor. Journal of neurophysiology 2015, 113 (4), 1249-59.

20. Ng, D. N.; Fromherz, P., Genetic Targeting of a VoltageSensitive Dye by Enzymatic Activation of Phosphonooxymethylammonium Derivative. ACS Chemical Biology 2011, 6 (5), 444-451.

21. Sundukova, M.; Prifti, E.; Bucci, A.; Kirillova, K.; Serrao, J.; Reymond, L.; Umebayashi, M.; Hovius, R.; Riezman, H.; Johnsson, K.; Heppenstall, P. A., A Chemogenetic Approach for the Optical Monitoring of Voltage in Neurons. Angewandte Chemie (International ed. in English) 2019, 58 (8), 2341-2344.

22. Fiala, T.; Wang, J.; Dunn, M.; Šebej, P.; Choi, S. J.; Nwadibia, E. C.; Fialova, E.; Martinez, D. M.; Cheetham, C. E.; Fogle, K. J.; Palladino, M. J.; Freyberg, Z.; Sulzer, D.; Sames, D., Chemical Targeting of Voltage Sensitive Dyes to Specific Cells and Molecules in the Brain. Journal of the American Chemical Society 2020, 142 (20), 9285-9301.

23. Abdelfattah, A. S.; Kawashima, T.; Singh, A.; Novak, O.; Liu, H.; Shuai, Y.; Huang, Y.-C.; Campagnola, L.; Seeman, S. C.; Yu, J.; Zheng, J.; Grimm, J. B.; Patel, R.; Friedrich, J.; Mensh, B. D.; Paninski, L.; Macklin, J. J.; Murphy, G. J.; Podgorski, K.; Lin, B.-J.; Chen, T.-W.; Turner, G. C.; Liu, Z.; Koyama, M.; Svoboda, K.; Ahrens, M. B.; Lavis,
L. D.; Schreiter, E. R., Bright and photostable chemigenetic indicators for extended in vivo voltage imaging. Science (New York, N.Y.) 2019, 365 (6454), 699-704.

24. Huang, Y.-L.; Walker, A. S.; Miller, E. W., A Photostable Silicon Rhodamine Platform for Optical Voltage Sensing. Journal of the American Chemical Society 2015, 137 (33), 10767-10776.

25. Ginebaugh, S. P.; Cyphers, E. D.; Lanka, V.; Ortiz, G.; Miller, E. W.; Laghaei, R.; Meriney, S. D., The Frog Motor Nerve Terminal Has Very Brief Action Potentials and Three Electrical Regions Predicted to Differentially Control Transmitter Release. Journal of Neuroscience 2020, 40 (18), 3504-3516.

26. Klimas, A.; Ortiz, G.; Boggess, S. C.; Miller, E. W.; Entcheva, E., Multimodal on-axis platform for all-optical electrophysiology with near-infrared probes in human stem-cell-derived cardiomyocytes. Progress in Biophysics \& Molecular Biology 2020, 154, 62-70.

27. McNamara, H. M.; Dodson, S.; Huang, Y. L.; Miller, E. W.; Sandstede, B.; Cohen, A. E., Geometry-Dependent Arrhythmias in Electrically Excitable Tissues. Cell Systems 2018, 7 (4), 359-+.

28. McNamara, H. M.; Salegame, R.; Al Tanoury, Z.; Xu, H. T.; Begum, S.; Ortiz, G.; Pourquie, O.; Cohen, A. E., Bioelectrical domain walls in homogeneous tissues. Nature Physics 2o2o, 16 (3), 357-+.

29. Streit, J.; Kleinlogel, S., Dynamic all-optical drug screening on cardiac voltage-gated ion channels. Scientific Reports 2o18, 8.

30. Lee-Montiel, F. T.; Laemmle, A.; Dumont, L.; Lee, C. S.; Huebsch, N.; Charwat, V.; Okochi, H.; Hancock, M. J.; Siemons, B.; Bogess, S.; Goswami, I.; Miller, E. W.; Willenbring, H.; Healy, K., Integrated hiPSC-based liver and heart microphysiological systems predict unsafe drug-drug interaction. bioRxiv 2020, 2020.05.24.112771.

31. Huebsch, N.; Charrez, B.; Siemons, B.; Boggess, S. C.; Wall, S.; Charwat, V.; Jæger, K. H.; Lee Montiel, F. T.; Jeffreys, N. C.; Deveshwar, N.; Edwards, A.; Serrano, J.; Snuderl, M.; Stahl, A.; Tveito, A.; Miller, E. W.; Healy, K. E., Metabolically-Driven Maturation of hiPSC-Cell Derived Heart-on-a-Chip. bioRxiv 2o18, 485169.

32. Deal, P. E.; Kulkarni, R. U.; Al-Abdullatif, S. H.; Miller, E. W., Isomerically Pure Tetramethylrhodamine Voltage Reporters. JAm Chem Soc 2016, 138 (29), 9085-8.

33. Deal, P. E.; Liu, P.; Al-Abdullatif, S. H.; Muller, V. R.; Shamardani, K.; Adesnik, H.; Miller, E. W., Covalently Tethered Rhodamine Voltage Reporters for High Speed Functional Imaging in Brain Tissue. Journal of the American Chemical Society 2020, 142 (1), 614-622.

34. Wang, B.; Chai, X.; Zhu, W.; Wang, T.; Wu, Q., A general approach to spirolactonized Si-rhodamines. Chemical Communications 2014, 50 (92), 14374-14377.

35. Kozma, E.; Estrada Girona, G.; Paci, G.; Lemke, E. A.; Kele, P., Bioorthogonal double-fluorogenic siliconrhodamine probes for intracellular super-resolution microscopy. Chemical Communications 2017, 53, 6696-6699.

36. Woodford, C. R.; Frady, E. P.; Smith, R. S.; Morey, B.; Canzi, G.; Palida, S. F.; Araneda, R. C.; Kristan, W. B.; Kubiak, C. P.; Miller, E. W.; Tsien, R. Y., Improved PeT Molecules for Optically Sensing Voltage in Neurons. Journal of the American Chemical Society 2015, 137 (5), 1817-1824.

37. Tabata, H.; Nakajima, K., Efficient in utero gene transfer system to the developing mouse brain using electroporation: visualization of neuronal migration in the developing cortex. Neuroscience 2001, 103 (4), 865-872.

38. Liu, P.; Grenier, V.; Hong, W.; Muller, V. R.; Miller, E. W., Fluorogenic Targeting of Voltage-Sensitive Dyes to Neurons. Journal of the American Chemical Society 2017, 139 (48), 17334-17340.

39. Ortiz, G.; Liu, P.; Naing, S. H. H.; Muller, V. R.; Miller, E. W., Synthesis of Sulfonated Carbofluoresceins for Voltage Imaging. Journal of the American Chemical Society 2019, 141 (16), 6631-6638.

40. Grenier, V.; Daws, B. R.; Liu, P.; Miller, E. W., Spying on Neuronal Membrane Potential with Genetically Targetable Voltage Indicators. Journal of the American Chemical Society 2019, 141 (3), 1349-1358. 
Authors are required to submit a graphic entry for the Table of Contents (TOC) that, in conjunction with the manuscript title, should give the reader a representative idea of one of the following: A key structure, reaction, equation, concept, or theorem, etc., that is discussed in the manuscript. Consult the journal's Instructions for Authors for TOC graphic specifications.

A silicon-rhodamine chemical-genetic hybrid for far red voltage imaging from defined neurons in brain slice

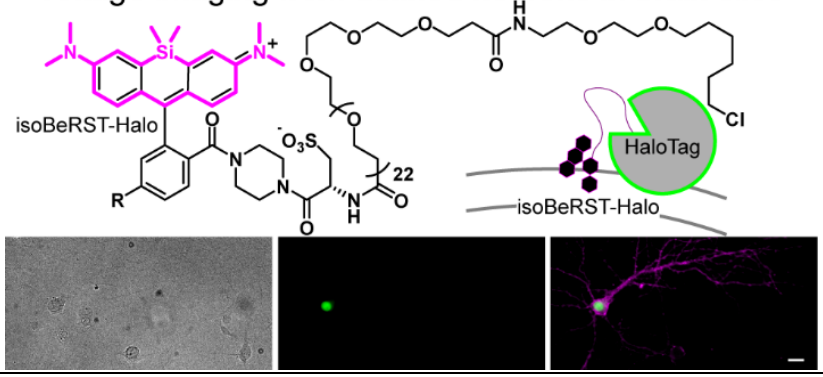

